\title{
On Semi-Uniformity, Quasi-Uniformity, Local Uniformity and Uniformity
}

\author{
Dr. Ranjan Kumar Singh \\ Deptt. of Mathematics RRM Campus \\ Janakpur (Nepal)
}

\begin{abstract}
In this paper we shall study the notions of semi-uniformity, quasi-uniformity, local uniformity and uniformity and we can establish some relations among them. Here it can be shown that a uniform space has always a base of symmetric vicinities.
\end{abstract}

Keywords:- Uniformity, Vicinity, Symmetric, Diagonal Filter.

\section{INTRODUCTION}

If the symmetry, condition in the definition of a pseudometric is deleted, the notion of a quasi-pseudometric is obtained. Asymmetric distance functions already occurs in the work of hausdorff in the beginning of the twentieth century when in his book on set-theory he discusses what is know called the hausdroff metric of a metric space.

A family of pseudo-metrices on a set generates uniformity. Similarly, a family of quasi-pseudometrices on a set generates a quasi- uniformity.

In 1937 Weil- published his booklet on uniformites, which is now usually considered as the beginning of the modern-theory of uniformities. Three years later Tukey suggested an approach to uniformities via uniform coverings. The study of quasi-uniformities started in 1948 with Nachbin's investigations on uniform pre-ordered preorder is given by the intersection of the entourages of a (filter) quasi-uniformity $u$ and sup-uniformity $u v u^{-1}$.

\section{NOTIONS OF SEMI-UNIFORMITY, QUASI- UNIFORMITY, LOCAL UNIFORMITY AND UNIFORMITY}

Definition :

Let $X$ be a non-empty set and let $\Delta=\{(x, x)\{x \in X\}$ be the 'diagonal'. A subset $A \subset X \times X$ is said to be 'symmetric' if $A=A^{-1}$, where $A^{-1}=\{(x, y) \in X \times X:(y, x) \in A\}$. For $A, B \subset X \times X$ we define $A, B=[(x, y):(x, z) \in A]$ and $(z, y) \in B$ for some $z \in X\}$
The set $\mathbf{A} . \mathbf{A}$ is also written as $\mathbf{A}^{2}$

If $A, B, C \subset X \times X$ then it is easy to see that

(i) $\quad A \cdot \Delta=\Delta \cdot A=A$;

(ii) $\quad A .(B, C)=(A \cdot B) \cdot C$;

(iii) $\quad(A . B)^{-1}=B^{-1} \cdot A^{-1}$

(iv) $\quad A^{n+1}=A^{n} \cdot A$ and $A^{n} \cdot A^{m}=A^{n+m}$;

(v) $\quad\left(A^{n}\right)^{-1}=\left(A^{-1}\right)^{n}$

(vi) $\quad A \subset B=>A^{-1} \subset B^{-1}$ and $A^{n} \subset B^{n}$

(vii) If $\mathrm{A}$ is symmetric so is $\mathrm{A}^{\mathrm{n}}$ for each positive interger n.

Proposition :

Let $\mathrm{B}$ be a filter base on $X \times X$ such that for each $U \in B$ we have

(i) $\quad \Delta \subseteq U$ (ii) $V^{-1} \subset U$ for some $V \in B$ and (iii) $V^{2} \subset U$ for some $V \in B$. Then the filter $\mathrm{F}$ (B) determined by B satisfies

(i) $\quad \Delta \subseteq U$ for all $U \in F(B)$

(ii), $U^{1} \in F(B)$ for all $U \in F(B)$;

(iii)' For all $U \in F(B)$, there is a $V \in F(B)$ such that $V^{2} \subset U$.

Conversely, suppose $\mathrm{F}$ is a filter on $X \times X$ satisfying (i)' to (iii)'.

Then the conditions (i) to (iii) are satisfied by every filterbase $\mathrm{B}$ which determines $\mathrm{F}$.

Proof:

(i)' is obvious for if $U \in F(B)$, then $U \geq U_{1}$ for some $U_{1} \in B$ and by (i) we have $\Delta \subseteq U_{1} \subset U$. To prove (ii)' and (iii)', consider any $U \in F(B)$. Then $W \subset U$ for some $W \in B$. Using (ii) we have a $V \in B \subset F(B)$ satisfying

$V^{1} \subset W \subset U$ and so $V \subset U^{1} ;$ that is $U^{1} \in F(B)$. Also by (iii) there is a $V \in B$ such that $V^{2} \subset W \subset U$ i.e. $V^{2} \subset U$. 
Conversely, suppose that $\mathrm{F}$ is filter on $X \times X$ which satisfies conditions (i)' to (iii)' and let $\mathrm{B}$ be a filter base on $X \times X$ which determine $\mathrm{F}$. since $B \subset F(B)$, condition (i) is satisfied. If $U \in B$ then $U^{-1} \in F(B)$ and $V \subset U^{-1}$ for some $V \in B$. Thus $V^{-1} \subset U$ and condition (ii)' is satisfied. Finally, let $U \in B \subseteq F(B)$ and let $W^{2} \subset U$. Since $W \in F(B)$, one has $V \subset W$ for some $V \in B$. In particular, we have $V^{2} \subset W^{2} \subset U$. Hence condition (iii)' is satisfied. This completes that proof.

\section{Definition :}

Let $\mathrm{X}$ be a non-empty set.

A filter $\mathcal{U}$ of subsets of $X \times X$ is said to be a

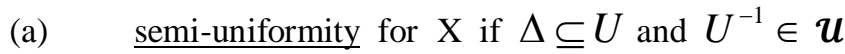
for all $U \in \mathcal{u}$,

(b) quasi-uniformity for $\mathrm{X}$ if $\Delta \subseteq U$ and there exists a $V \in \mathcal{U}$ such that $V^{2} \subset U$ for all $U \in \mathcal{u}$,

(c) Uniformity for $\mathrm{X}$ if $\boldsymbol{U}$ is both a semiuniformity and a quasi-uniformity.

(d) Local uniformity for $\mathrm{X}$ if it is semi-uniformity and for each $U \in \mathcal{U}$ and $x \in X$, there exists a $V \in \mathcal{U}$ such that $V^{2}[x] \subset U[X], \boldsymbol{u}$ is said to be Hausdorff (or separated) if $\bigcap\{U: U \in u\}=\Delta$. The members $U \in \mathcal{U}$ are called the Vicinities. Moreover, $x, y \in X$ are called Uclose (or close of order $\mathrm{U}$ ) if $(x, y) \in U$ (where $U \in \boldsymbol{u}$ ).

\section{Definition :}

Uniform space: A uniform space is a pair $(\mathrm{X}, \boldsymbol{U})$ where $\mathrm{X}$ is a non-empty set and $\boldsymbol{U}$ is a uniformity for $\mathrm{X}$, i.e. $\mathcal{u}$ is a filter on $X \times X$ satisfying the following conditions:

$\left[V_{1}\right]$ Every $U \in \mathcal{U}$ contains the diagonal $\Delta$;

$\left[V_{2}\right]$ If $\boldsymbol{U} \in \boldsymbol{u}$ then $U^{-1} \in \boldsymbol{u}$

$\left[V_{3}\right]$ for each $U \in \mathcal{u}$ there exists a $V \in \mathcal{u}$ with $V^{2} \subset U$

\section{COMPARISON OF UNIFORMITIES :}

\section{$>$ Definition :}

If $\boldsymbol{u}_{\mathbf{1}}$ and $\boldsymbol{u}_{\mathbf{2}}$ are both uniformities for a non-empty set $\mathrm{X}$, we say that $\boldsymbol{U}_{1}$ is weaker or coarser than $\boldsymbol{u}_{\mathbf{2}}$ (and $\boldsymbol{u}_{\mathbf{2}}$ if finer or stronger than $\boldsymbol{U}_{1}$ ) if $\boldsymbol{U}_{\mathbf{1}} \subseteq \mathcal{U}_{2}$. that is, if every vicinity for $\boldsymbol{U}_{1}$ is also a vicinity for $\boldsymbol{U}_{2}$.

\section{$>$ Definition:}

A base for a uniformity $\boldsymbol{U}$ on $\mathrm{X}$ is a filterbase $\beta$ such that $U \in \mathcal{U}$ iff $U$ contains some $B \in \beta$.
Proof (6.2) :

A uniform space $(\mathrm{X}, \boldsymbol{U})$ has always a base of symmetric vicinities.

Proof :

Let $\beta=$ the family of all symmetric vicinities i.e. those with $U=U^{-1}$

For $\boldsymbol{U} \in \boldsymbol{U}$. Then $\beta$ is a base for $\boldsymbol{U}$ since the symmetric vicinity $U \cap U^{-1}$ is contained in the vicinity $U$ for each $U \in \mathcal{u}$.

\section{$>$ Definition :}

Let $\beta_{1}$ and $\beta_{2}$ be two bases for a uniformity $\mathrm{U}$ on a non-empty set $\mathrm{X}$ we say that $\beta_{1}$ and $\beta_{2}$ are equivalent if for each $B_{1} \in \beta_{1}$ there exists a $B_{2} \in \beta_{2}$ such that $B_{2} \subseteq B_{1}$ and for each $B_{2}{ }^{\prime} \in \beta_{2}$ there exists $B_{1}{ }^{\prime} \in \beta_{1}$ such that $B_{1}{ }^{\prime} \subseteq B_{2}{ }^{\prime}$.

\section{Example (1) :}

Given a non-empty set $X$, the indiscrete uniformity $\mathcal{u}_{1}=X \times X$ is the weakest uniformity for $\mathrm{X}$ and the discrete uniformity $\mathcal{u}_{D}=\{U \subset X \times X ; \Delta \subseteq U\}$ is the strongest uniformity for $\mathrm{X}$. We note that $\{\Delta\}$ is a base for discrete uniformity for $\mathrm{X}$ where as $\{X \times X\}$ is a base for indiscrete uniformity for $\mathrm{X}$.

\section{CONCLUSION}

Hence, A uniform space $(\mathrm{X}, \boldsymbol{U})$ has always a base of symmetric vicinities.

\section{REFERENCES}

[1]. Munkres, J.R. : $\quad$ Topology, A first course, Prentice-Hall of India, 1984.

[2]. Murdeshwar M.G. : 'Quasi-uniform topological spaces', Sec. A, Vol. 2, no. 4, Ealbner, New York, 1966.

[3]. Nagata, J. I. : : 'Modern general topology', Third Revised edition, North Holland, 1985.

[4]. Pervin, W. J. : 'Uniformities of nhd axioms', Math, Ann. 147 (1962), 313-315.

[5]. Pachl, Jan : Uniform spaces \& measures, springer Scinece\& Business media NewYork 2013. 